\title{
Association Between Fear of Falling and Frailty in Community-Dwelling Older Adults: A Systematic Review
}

\author{
Larissa Franciny de Souza', Jaquelini Betta Canever', Bruno de Souza Moreira (D) ${ }^{2}$,

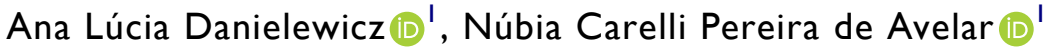 \\ 'Laboratory of Aging, Resources and Rheumatology, Department of Health Sciences, Federal University of Santa Catarina, Araranguá, Santa Catarina, \\ Brazil; ${ }^{2}$ René Rachou Institute, Oswaldo Cruz Foundation (Fiocruz Minas), Belo Horizonte, Minas Gerais, Brazil
}

Correspondence: Bruno de Souza Moreira, René Rachou Institute, Oswaldo Cruz Foundation (Fiocruz Minas), Augusto de Lima Avenue I7I5, Barro Preto, Belo Horizonte, Minas Gerais, 30190-002, Brazil, Tel +55 3I 3349-7707, Email brunosouzamoreira@gmail.com

Background/Objective: Fear of falling (FoF) and frailty are common problems in older adults. FoF can lead to self-imposed restriction of activities and then further decline in physical capacities that predispose older adults to frailty. Evaluating the association of these two geriatric syndromes may be the first step for understanding their complex relationship and might ultimately lead to establishing therapeutic goals and guiding treatments for older adults with frailty. This systematic review was conducted to provide evidence regarding the association between FoF and frailty.

Methods: All the articles that provided information on the association between FoF and frailty were selected from PubMed, Scopus, CINAHL, and EMBASE in search of relevant papers. Articles reporting information on the association between FoF (exposure) and frailty (outcome), with older adults (age $\geq 60$ years) living in the community (ie, living either at home or in places of residence that do not provide nursing care or rehabilitation) were included. Only original articles with observational design (cross-sectional or longitudinal/cohort) were included. The methodological quality of included articles was evaluated independently by the two assessors through the Newcastle-Ottawa Scale (NOS) and the Joanna Briggs Institute (JBI) critical appraisal checklist for longitudinal and crosssectional studies, respectively.

Results: The initial searches found 4,342 articles, of which 10 articles were included in this review: 7 cross-sectional and 2 longitudinal studies, and 1 study with cross-sectional and longitudinal analyses. The total sample was composed of 6,294 community-dwelling older adults (61.8\% women). Among the longitudinal studies, adjusted odds ratios ranged from $1.18(95 \% \mathrm{CI}=1.02 ; 1.36)$ to $9.87(95 \% \mathrm{CI}=5.22$; 18.68), while the adjusted odds ratios of the cross-sectional studies ranged from $1.04(95 \% \mathrm{CI}=1.02 ; 1.07)$ to $7.16(95 \% \mathrm{CI}=2.34 ; 21.89)$. Conclusion: FoF increases the risk of frailty in community-dwelling older adults. The knowledge of this association is of utmost importance in clinical practice, since it can help health professionals in the development of rehabilitation, prevention, and health promotion protocols. In addition, these findings can contribute to the development of public health policies and actions aimed at reducing the FoF and consequently the frailty.

Prospero: CRD42021276775.

Keywords: fear of falling, frailty, older adults, systematic review, geriatric syndromes

\section{Introduction}

Frailty is a serious public health problem in aging societies, affecting between $4.0 \%$ and $59.1 \%$ of community-dwelling older adults aged 65 years and over from the United States, Canada, Europe, Taiwan, and Australia. ${ }^{1}$ In low- and middleincome countries, frailty prevalence ranges from $3.9 \%$ to $51.4 \%$ in individuals aged 60 years and over living in the community. ${ }^{2}$ This complex and multidimensional syndrome is understood as a state of higher vulnerability to endogenous and exogenous stressors (eg, infection, injury, surgery, or psychosocial distress), as a result of age-related declines in function and reserve of various physiological systems, increasing the probability of adverse health outcomes. ${ }^{3}$ A recent 
systematic review of 29 prospective studies showed that frail individuals had a significantly higher risk of falls, bone fractures, disability, dementia, hospitalization, and death. ${ }^{4}$

Some experts in geriatrics have also conceptualized frailty as a pre-disability state; however, it can co-exist with disability. ${ }^{5,6}$ Other researchers consider frailty as an indicator of functional decline that represents a life stage between autonomous living and death. ${ }^{4,7}$ Sociodemographic (advanced age, female sex, low education level, low income, living alone, and loneliness), clinical (multimorbidity, obesity, malnutrition, impaired cognition, depressive symptoms, and polypharmacy), lifestyle (physical inactivity, low protein consumption, smoking, and increased alcohol intake), and biological factors (inflammation, endocrine problems, and micronutrient deficits) are associated with onset or progression of frailty. ${ }^{8,9}$ Studies have shown that all older adults are at risk of developing frailty; however, the risk is eminently greater among those with comorbidities, low socioeconomic position, poor diet, and sedentary lifestyles. ${ }^{8}$

Fear of falling (FoF) is another common problem in the older population. One of the concepts of FoF refers to low perceived self-efficacy or confidence at avoiding falls during essential, nonhazardous activities of daily living. ${ }^{10}$ Prevalence rates for FoF in community-dwelling older adults range from $21.0 \%$ to $85.0 \%$ among fallers, and $33.0 \%$ to $46.0 \%$ among those with no history of falls. ${ }^{11}$ FoF can be considered a protective response because it allows a person to be more cautious about her/his surroundings. However, when irrational and exaggerated, FoF can affect the person's physical and psychosocial well-being due to restriction or avoidance of activities. ${ }^{12}$ In the long term, restriction of activities can lead to deconditioning, muscle weakness, and postural instability, which in turn can favor the occurrence of future falls. ${ }^{13}$ Moreover, FoF has been linked to other negative consequences including disability for performing basic activities of daily living, reduced social interactions, depression, poor health-related quality of life, and all-cause mortality. ${ }^{14-16}$

The hypothesis of the present study is that FoF would lead to self-imposed restriction of activities and, then further decline in physical capacities (eg, conditioning, muscle strength, and balance), predisposing older adults to frailty. In other words, FoF would be a risk factor for frailty. To date, to the best of our knowledge, no systematic review focusing on the association between FoF and frailty has been conducted. Evaluating the association of these two geriatric syndromes may be the first step for understanding their complex relationship and might ultimately lead to establishing therapeutic goals and guiding treatments for older adults with frailty. Furthermore, summarizing the results about this topic raises the evidence level beyond findings derived from single previous studies, which can provide guidance to further research. Therefore, the present study aimed to systematically review the available literature on the association of FoF with frailty in community-dwelling older adults.

\section{Methods}

\section{Protocol and Registration}

The protocol of the present study was registered in the International Prospective Register of Systematic Reviews PROSPERO (registration number CRD42021276775). This systematic review was conducted in accordance with the Preferred Reporting Items for Systematic Reviews and Meta-Analyses (PRISMA) recommendations.

\section{Definition of Variables}

Participants were classified according to their frailty status and FoF using any currently validated scale, physical tests, or measurements for identification of each condition.

\section{Search Strategy}

The searches were conducted in October 2021 in the PubMed, Scopus, CINAHL, and EMBASE databases (Appendix 1). The Medical Subject Headings (MeSH) were chosen, but when these could not be employed due to absence in the thesaurus or the excess of results inconsistent with the purpose of the study, the most common descriptors of articles with respect to the topic were used. In such cases, the search restriction was made to the descriptors, titles, and abstracts.

The search included as the first descriptor the participants (older adults OR older people OR elderly OR aged OR aging OR ageing OR older person). The second descriptor was FOF (fear-related activity restriction OR fear of falls OR 
fear of falling OR fear OR fall-related OR self-efficacy OR balance confidence fear-associated OR related mobility restriction OR activity restriction OR fall-related efficacy OR falls-efficacy OR self-confidence). The third one was frailty (frail*).

\section{Study Selection and Data Extraction}

The selection of articles was carried out by two reviewers (L.F.S. and J.B.C.), independently and in two stages. First, the titles and abstracts of all identified articles were screened. Next, the articles were read in full. A third assessor review the data extraction (N.C.P.A.), and any disagreement was resolved through consensus.

Duplicate references in two or more bibliographic databases were excluded with the help of the bibliographic management program (Mendeley) and subsequently checked manually. The references lists of all selected articles were explored in order to identify other articles relevant to the objective of the review that had not been found by the electronic search.

The data of the selected articles were extracted and entered independently by the first two reviewers in a spreadsheet in the program Microsoft Excel 2010, and then compared for pairing and possible corrections. Information with regard to title, authors, year of publication, country as well as data on the study design, study sample, outcome, exposure, control variables, applied statistical analyses, and results of the investigated associations (magnitudes and respective 95\% confidence intervals $-95 \% \mathrm{CI}$ ) were collected.

\section{Eligibility Criteria}

Articles reporting information on the association between FoF (exposure) and frailty (outcome), with older adults (age $\geq$ 60 years) living in the community (ie, living either at home or in places of residence that do not provide nursing care or rehabilitation) were included. Only original articles with observational design (cross-sectional or longitudinal/cohort) were included. No restrictions were done for language or year of publication. Theses, dissertations, and monographs were not included.

Articles that did not define frailty or assessed it only with a single symptom/measure (eg, only gait speed or grip strength), and did not report the association between FoF and frailty were excluded. Articles that included persons younger than 60 years, combined populations (ie, community-dwelling older adults and those receiving nursing care or rehabilitation), conducted with populations with a specific health condition (eg, stroke or hip fracture), used FoF and frailty as confounding variables, or did not perform quantitative analyses were also excluded.

\section{Assessment of Risk of Bias}

The methodological quality of included articles was evaluated independently by the two assessors (L.F.S. and J.B.C.) through the Newcastle-Ottawa Scale (NOS) and the Joanna Briggs Institute (JBI) critical appraisal checklist for longitudinal and cross-sectional studies, respectively. The NOS scale has 8 items evaluating four dimensions, including sample selection, sample representativeness, comparability, and outcome assessment. Each item is awarded a maximum of one star except for the domain of "comparability", which is awarded a maximum of two stars. More stars indicate a higher quality. ${ }^{17}$

The JBI critical appraisal checklist contains 8 items assessing sample selection criteria, subject description, exposure measurement, subject condition measurement, confounding factors identification, confounding factors control, outcome assessment, and statistical analysis. Each item is categorized as yes, no, unclear, or not applicable. ${ }^{18}$ Any disagreement in quality assessment was resolved through consensus. For both study designs, articles scoring $>7$ are considered at low risk of bias, scores of 5-7 indicate a moderate risk of bias, and scores of $<5$ indicate a high risk of bias.

\section{Results}

The initial searches in the electronic databases identified 4,342 articles for review. A total of 4,271 titles was screened after 71 duplicates were removed. Then, 4,254 articles were excluded based on titles and abstracts, and 17 full-text articles were reviewed for eligibility. In the end, 10 articles met the eligibility criteria for literature synthesis (Figure 1). 


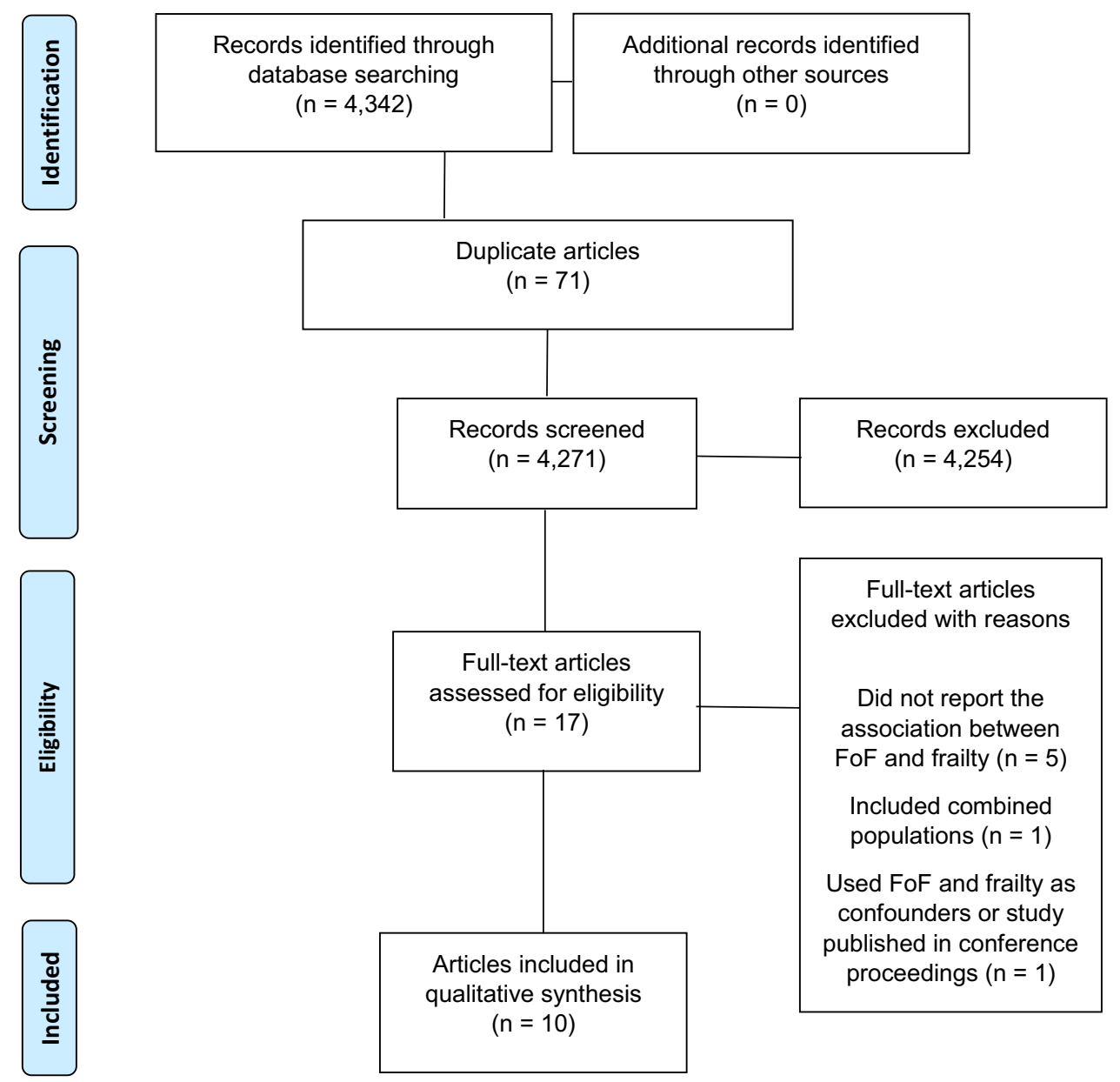

Figure I Study flowchart. Adapted from Page MJ, McKenzie JE, Bossuyt PM, et al. The PRISMA 2020 statement: an updated guideline for reporting systematic reviews. BMJ. 202I;372:n7l. ${ }^{41}$

Table 1 shows the characteristics of the included articles in the systematic review. Although there was no restriction regarding the year of publication of the articles, it is noted that $70 \%(\mathrm{n}=7)$ of the articles were published in the last two years [2021: three articles $(30 \%){ }^{19-21}$ 2020: four articles $\left.(40 \%)^{22-25}\right] ; 2015,2011,1994$ : one article $(10 \%)$ for each year. ${ }^{26-28}$ Four studies were conducted in high income countries [Spain $(\mathrm{n}=1),{ }^{19}$ Japan $(\mathrm{n}=1),{ }^{24}$ Singapore $(\mathrm{n}=1),{ }^{22}$ and United States $(n=1)],{ }^{28}$ five in upper-middle-income countries [(Brazil $(n=1),{ }^{26}$ Turkey $(n=2),{ }^{25,27}$ Taiwan $(n=1),{ }^{21}$ China $(\mathrm{n}=1)],{ }^{20}$ and one study was conducted with older adults living in high (Canada) and upper-middle-income countries (Albania, Colombia, and Brazil). ${ }^{23}$

Seven studies had a cross-sectional design, ${ }^{19-22,25-27}$ two were longitudinal studies, ${ }^{23,28}$ and one study had longitudinal and cross-sectional analyses. ${ }^{24}$ The sample size of the included articles ranged from 113 to 1,434 participants, totaling 6,294 older adults. The participants' age ranged from 64 to $>81$ years $(73.11 \pm 2.29$ years). Of the total sample, $61.8 \%$ were women and $38.2 \%$ were men. Overall, half of the studies considered two frailty groups (pre-frail and frail), ${ }^{20,22,23,25,27}$ one study considered only isolated frailty group (frail), ${ }^{24}$ and four studies did not provide any division according to frailty status. ${ }^{19,21,26,28}$

As regards the frailty identification, one-half of studies ${ }^{22,23,25-27}$ used the Fried et al's scale. ${ }^{29}$ One study ${ }^{24}$ used at least one modified criterion of Fried et al's scale. ${ }^{29}$ In addition, four studies used other tools to assess frailty: Short Physical Performance Battery, ${ }^{19}$ Index Study of Osteoporotic Fractures, ${ }^{21}$ Tilburg Frailty Indicator, ${ }^{20}$ and physical test and measures. ${ }^{28}$ Regarding the FoF assessment, four studies used the Falls Efficacy Scale-International (FES-I), ${ }^{20,21,23,26}$ three studies used the Short FES-I, ${ }^{19,21,24}$ and four studies used a self-report question. ${ }^{22,25,27,28}$ 


\begin{tabular}{|c|c|c|c|c|c|c|c|c|c|c|c|c|c|c|}
\hline \multirow[t]{2}{*}{ Study } & \multirow[t]{2}{*}{ Country } & \multirow{2}{*}{$\begin{array}{l}\text { Study } \\
\text { Design }\end{array}$} & \multirow{2}{*}{$\begin{array}{c}\text { Total } \\
\text { Population }\end{array}$} & \multirow{2}{*}{$\begin{array}{c}\text { Sample } \\
\text { Characteristics } \\
\text { (Age and Sex) }\end{array}$} & \multirow{2}{*}{$\begin{array}{l}\text { Frailty } \\
\text { Criterion }\end{array}$} & \multicolumn{2}{|c|}{ Prevalence } & \multirow{2}{*}{$\begin{array}{l}\text { Fof Criterion } \\
\text { (Categorization) }\end{array}$} & \multicolumn{2}{|c|}{ Prevalence } & \multirow{2}{*}{$\begin{array}{l}\text { Adjustment } \\
\text { Variables }\end{array}$} & \multirow{2}{*}{$\begin{array}{l}\text { Statistical } \\
\text { Methods }\end{array}$} & \multirow{2}{*}{$\begin{array}{l}\text { Unadjusted } \\
\text { OR (95\% } \\
\text { CI) }\end{array}$} & \multirow[t]{2}{*}{ Adjusted OR $(95 \% \mathrm{Cl})$} \\
\hline & & & & & & \begin{tabular}{|l|} 
Frail \\
\end{tabular} & $\begin{array}{l}\text { Pre- } \\
\text { Frail }\end{array}$ & & $\begin{array}{l}\text { High } \\
\text { FoF }\end{array}$ & $\begin{array}{l}\text { Low } \\
\text { FoF }\end{array}$ & & & & \\
\hline $\begin{array}{l}\text { Öztürk } \\
\text { et al. } \\
(2020)^{25}\end{array}$ & Turkey & $\begin{array}{c}\text { Cross- } \\
\text { sectional }\end{array}$ & 1,021 & $\begin{array}{c}\text { Age } 74.9 \pm 6.9 \\
\text { Female } 693 \\
\text { Male } 328\end{array}$ & $\begin{array}{l}\text { Fried et al's } \\
\text { scale }\end{array}$ & $19.20 \%$ & $42.10 \%$ & $\begin{array}{l}\text { Self-reported } \\
\text { question } \\
\text { "Are you afraid of } \\
\text { falling?" }\end{array}$ & . & $44.60 \%$ & . & $\begin{array}{c}\text { Multivariate } \\
\text { logistic } \\
\text { regression } \\
\text { analysis }\end{array}$ & $0.8(0.4 ; 1.5)$ & . \\
\hline $\begin{array}{l}\text { Alcolea- } \\
\text { Ruiz et al. } \\
(2021)^{19}\end{array}$ & Spain & $\begin{array}{c}\text { Cross- } \\
\text { sectional }\end{array}$ & 189 & $\begin{array}{c}\text { Age } 77(73.0- \\
80.0) \\
\text { Female } 117 \\
\text { Male } 72\end{array}$ & $\begin{array}{c}\text { Short Physical } \\
\text { Performance } \\
\text { Battery } \\
\text { (SPPB) }\end{array}$ & - & - & Short FES-I & - & $42.90 \%$ & - & $\begin{array}{c}\text { Binary and } \\
\text { multivariate } \\
\text { logistic } \\
\text { regression } \\
\text { analysis }\end{array}$ & - & $1.7(1.3 ; 2.2)$ \\
\hline $\begin{array}{l}\text { Kuo et al. } \\
(2021)^{21}\end{array}$ & Taivan & $\begin{array}{c}\text { Cross- } \\
\text { sectional }\end{array}$ & 751 & $\begin{array}{c}\text { Age } 73.6 \pm 6.6 \\
\text { Female } 477 \\
\text { Male } 274\end{array}$ & $\begin{array}{l}\text { Index Study of } \\
\text { Osteoporotic } \\
\text { Fractures } \\
\text { (SOF) }\end{array}$ & - & - & $\begin{array}{l}\text { Short FES-I and } \\
\text { FES-I }\end{array}$ & - & - & $\begin{array}{l}\text { Sex, age, marital } \\
\text { status, educational } \\
\text { attainment, working } \\
\text { status, living } \\
\text { arrangement, body } \\
\text { mass index, } \\
\text { smoking, and } \\
\text { drinking status }\end{array}$ & $\begin{array}{l}\text { Multivariate } \\
\text { logistic } \\
\text { regression } \\
\text { analysis }\end{array}$ & - & 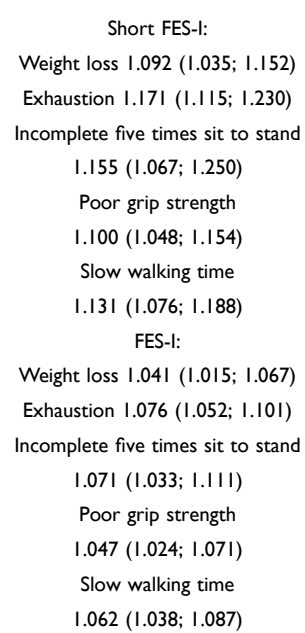 \\
\hline $\begin{array}{l}\text { Qin et al. } \\
(2021)^{20}\end{array}$ & China & $\begin{array}{c}\text { Cross- } \\
\text { sectional }\end{array}$ & 158 & $\begin{array}{c}\text { Age } 71.63 \pm 7.98 \\
\text { Female } 66 \\
\text { Male } 92\end{array}$ & $\begin{array}{l}\text { Tilburg Frailty } \\
\text { Indicator }\end{array}$ & $60.10 \%$ & $36.90 \%$ & FES-I & & $81.0 \%$ & $\begin{array}{c}\text { Age, hospitalizations } \\
\text { in the past year, fall } \\
\text { history in the past } 6 \\
\text { months, and the } \\
\text { number of chronic } \\
\text { diseases }\end{array}$ & $\begin{array}{c}\text { Binary } \\
\text { logistic } \\
\text { regression } \\
\text { analysis }\end{array}$ & & $7.160(2.342 ; 21.894)$ \\
\hline
\end{tabular}


Table I (Continued).

\begin{tabular}{|c|c|c|c|c|c|c|c|c|c|c|c|c|c|c|}
\hline \multirow[t]{2}{*}{ Study } & \multirow[t]{2}{*}{ Country } & \multirow{2}{*}{$\begin{array}{l}\text { Study } \\
\text { Design }\end{array}$} & \multirow{2}{*}{$\begin{array}{c}\text { Total } \\
\text { Population }\end{array}$} & \multirow{2}{*}{$\begin{array}{c}\text { Sample } \\
\text { Characteristics } \\
\text { (Age and Sex) }\end{array}$} & \multirow{2}{*}{$\begin{array}{l}\text { Frailty } \\
\text { Criterion }\end{array}$} & \multicolumn{2}{|c|}{ Prevalence } & \multirow{2}{*}{$\begin{array}{l}\text { FoF Criterion } \\
\text { (Categorization) }\end{array}$} & \multicolumn{2}{|c|}{ Prevalence } & \multirow{2}{*}{$\begin{array}{l}\text { Adjustment } \\
\text { Variables }\end{array}$} & \multirow{2}{*}{$\begin{array}{l}\text { Statistical } \\
\text { Methods }\end{array}$} & \multirow{2}{*}{$\begin{array}{c}\text { Unadjusted } \\
\text { OR (95\% } \\
\text { Cl) }\end{array}$} & \multirow[t]{2}{*}{ Adjusted OR $(95 \% \mathrm{Cl})$} \\
\hline & & & & & & Frail & $\begin{array}{l}\text { Pre- } \\
\text { Frail }\end{array}$ & & $\begin{array}{l}\text { High } \\
\text { FoF }\end{array}$ & $\begin{array}{l}\text { Low } \\
\text { FoF }\end{array}$ & & & & \\
\hline $\begin{array}{l}\text { Kamide } \\
\text { et al. } \\
(2020)^{24}\end{array}$ & Japan & $\begin{array}{l}\text { Longitudinal } \\
\text { and cross- } \\
\text { sectional }\end{array}$ & 339 & $\begin{array}{c}\text { Age } 72.9 \pm \\
\quad 4.8 \\
\text { Female } 238 \\
\text { Male 101 }\end{array}$ & $\begin{array}{l}\text { Modified } \\
\text { Fried et al's } \\
\text { scale }\end{array}$ & $10.0 \%$ & - & Short FES-I & - & - & $\begin{array}{l}\text { Motor function } \\
\text { (handgrip test, } \\
\text { running time, sit and } \\
\text { stand up from a } \\
\text { chair test), } \\
\text { psychological } \\
\text { function (GDS-5), } \\
\text { anthropometry } \\
\text { (body mass index), } \\
\text { disability in daily life } \\
\text { activities, } \\
\text { comorbidities, } \\
\text { medications, history } \\
\text { of falls in the last } \\
\text { year, and pains. }\end{array}$ & $\begin{array}{l}\text { Multivariate } \\
\text { logistic } \\
\text { regression } \\
\text { analysis }\end{array}$ & & $\begin{array}{c}\text { Cross-sectional I.16 }(1.05 ; 1.29) \\
\text { Longitudinal I.18 }(1.02 ; 1.36)\end{array}$ \\
\hline $\begin{array}{l}\text { Merchant } \\
\text { et al. } \\
(2020)^{22}\end{array}$ & Singapore & $\begin{array}{l}\text { Cross- } \\
\text { sectional }\end{array}$ & 493 & $\begin{array}{c}\text { Age } 73 \pm 8 \\
\text { Female } 391 \\
\text { Male } 102\end{array}$ & $\begin{array}{l}\text { Fried et al's } \\
\text { scale }\end{array}$ & $3.40 \%$ & $47.90 \%$ & $\begin{array}{l}\text { Self-reported } \\
\text { question } \\
\text { "Are you afraid of } \\
\text { falling?" }\end{array}$ & - & $69.20 \%$ & - & $\begin{array}{l}\text { Bivariate } \\
\text { and } \\
\text { multivariate } \\
\text { logistic } \\
\text { regression } \\
\text { analysis }\end{array}$ & $\begin{array}{l}2.17(1.26 \\
3.73)\end{array}$ & - \\
\hline $\begin{array}{l}\text { Curcio } \\
\text { et al. } \\
(2020)^{23}\end{array}$ & $\begin{array}{l}\text { Canada, } \\
\text { Albania, } \\
\text { Brazil, } \\
\text { and } \\
\text { Colombia }\end{array}$ & Longitudinal & I,434 & $\begin{array}{l}\text { Age 64-69: } 808 \\
\text { Age 70-75: } 626 \\
\text { Female } 753 \\
\text { Male 68। }\end{array}$ & $\begin{array}{l}\text { Fried et al's } \\
\text { scale }\end{array}$ & $6.50 \%$ & $45.70 \%$ & FES-I & $75.20 \%$ & $24.80 \%$ & - & $\begin{array}{l}\text { Multivariate } \\
\text { logistic } \\
\text { regression } \\
\text { analysis }\end{array}$ & $\begin{array}{l}12.4(7.6 \\
20.1)\end{array}$ & - \\
\hline $\begin{array}{l}\text { Akin et al. } \\
(2015)^{27}\end{array}$ & Turkey & $\begin{array}{l}\text { Cross- } \\
\text { sectional }\end{array}$ & 906 & $\begin{array}{l}\text { Age } 71.5 \\
\quad \pm 5.6 \\
\text { Female } 459 \\
\text { Male } 447\end{array}$ & $\begin{array}{l}\text { Fried et al's } \\
\text { scale }\end{array}$ & $10.0 \%$ & $45.6 \%$ & $\begin{array}{l}\text { Self-reported } \\
\text { question } \\
\text { "FoF absent or } \\
\text { present during the } \\
\text { last year" }\end{array}$ & - & - & - & $\begin{array}{l}\text { Univariate } \\
\text { and } \\
\text { multivariate } \\
\text { logistic } \\
\text { regression } \\
\text { analysis }\end{array}$ & $\begin{array}{l}\text { I.452 (1.029; } \\
2.048)\end{array}$ & - \\
\hline
\end{tabular}




\begin{tabular}{|c|c|c|c|c|c|c|c|c|c|c|c|c|c|}
\hline $\begin{array}{l}\text { Dias et al. } \\
(2011)^{26}\end{array}$ & Brazil & $\begin{array}{l}\text { Cross- } \\
\text { sectional }\end{array}$ & 113 & $\begin{array}{c}\text { Age } 74.5 \pm 7.0 \\
\text { Female } 96 \\
\text { Male } 17\end{array}$ & $\begin{array}{l}\text { Fried et al's } \\
\text { scale }\end{array}$ & - & - & FES-I & & $28.32 \%$ & - & $\begin{array}{l}\text { ANOVA, } \\
\text { CHAID } \\
\text { method, } \\
\text { model } \\
\text { validation } \\
\text { verified } \\
\text { through } \\
\text { spliffolds } \\
\text { method }\end{array}$ & - \\
\hline $\begin{array}{l}\text { Arrken } \\
\text { et al. } \\
(1994)^{28}\end{array}$ & $\begin{array}{l}\text { United } \\
\text { States }\end{array}$ & Longitudinal & 890 & $\begin{array}{c}\text { Age 66-70: } 243 \\
\text { Age 7I-75: } 240 \\
\text { Age 76-80: } 203 \\
\text { Age >81: } 204 \\
\text { Female } 597 \\
\text { Male } 293\end{array}$ & $\begin{array}{l}\text { Physical test } \\
\text { and measures }\end{array}$ & - & - & $\begin{array}{l}\text { Self-reported } \\
\text { question } \\
\text { "At the present } \\
\text { time, are you very } \\
\text { fearful, somewhat } \\
\text { fearful or not } \\
\text { fearful that you } \\
\text { may fall } \\
\text { (again)?" }\end{array}$ & $46.61 \%$ & $53.39 \%$ & Age and sex & $\begin{array}{l}\text { Multivariate } \\
\text { logistic } \\
\text { regression } \\
\text { analysis }\end{array}$ & $\begin{array}{c}\text { Frailty impaired balance } \\
4.44(2.15 ; 9.17) \\
\text { Episode of near fall } \\
3.61(2.12 ; 6.13) \\
\text { Inability to walk } 10 \text { blocks } \\
3.48(1.99 ; 6.08) \\
\text { Requiring assistance to climb stairs } \\
\text { ambulation } \\
4.66(2.62 ; 8.30) \\
\text { Vision limiting 4.79 (2.09; } 11.01) \\
\text { Fair or poor self-rated health } \\
3.72(2.16 ; 6.40) \\
\text { Use of assistive device to ambulate } \\
9.87(5.22 ; 18.68)\end{array}$ \\
\hline
\end{tabular}

Abbreviations: FoF, Fear of Falling; FES-I, Falls Efficacy Scale-International; OR, odds ratio; ANOVA, analysis of variance; CHAID, Chi-Square Automatic Interaction Detection. 
Table 2 Quality Assessment of Longitudinal Studies ( $n=3)$ Using the Newcastle-Ottawa Scale (NOS)

\begin{tabular}{|c|c|c|c|c|c|c|c|c|c|}
\hline \multirow[t]{2}{*}{ Source } & \multicolumn{9}{|c|}{ Criteria } \\
\hline & $\begin{array}{c}\text { Representation } \\
\text { of the Exposed } \\
\text { Cohort }\end{array}$ & $\begin{array}{l}\text { Selection } \\
\text { of the } \\
\text { Non- } \\
\text { Exposed } \\
\text { Cohort }\end{array}$ & $\begin{array}{c}\text { Ascertainment } \\
\text { of Exposure }\end{array}$ & $\begin{array}{l}\text { Demonstration } \\
\text { That Outcome } \\
\text { of Interest Was } \\
\text { Not Present at } \\
\text { Start of Study }\end{array}$ & $\begin{array}{c}\text { Comparability } \\
\text { of Cohorts on } \\
\text { the Basis of } \\
\text { the Design or } \\
\text { Analysis }\end{array}$ & $\begin{array}{l}\text { Assessment } \\
\text { of Outcome }\end{array}$ & $\begin{array}{l}\text { Follow-Up } \\
\text { Was Long } \\
\text { Enough for } \\
\text { Outcomes } \\
\text { to Occur }\end{array}$ & $\begin{array}{c}\text { Adequacy } \\
\text { of Follow- } \\
\text { Up of } \\
\text { Cohorts }\end{array}$ & Total Score \\
\hline $\begin{array}{l}\text { Kamide } \\
\text { et al. } \\
(2020)^{24}\end{array}$ & $\star$ & & $\star$ & $\star$ & $\star$ & $\star$ & & & 5 \\
\hline $\begin{array}{l}\text { Curcio } \\
\text { et al. } \\
(2020)^{23}\end{array}$ & $\star$ & & $\star$ & $\star$ & & $\star$ & $\star$ & & 5 \\
\hline $\begin{array}{l}\text { Arfken } \\
\text { et al. } \\
(1994)^{28}\end{array}$ & $\star$ & & & $\star$ & $\star \star$ & & $\star$ & & 5 \\
\hline
\end{tabular}

The unadjusted odds ratio (OR) of the association between FoF (exposure) and frailty (outcome) was 12.4 (95\% $\mathrm{CI}=7.6 ; 20.1)^{23}$ and the adjusted ORs varied from $1.18(95 \% \mathrm{CI}=1.02 ; 1.36)^{24}$ to $9.87(95 \% \mathrm{CI}=5.22 ; 18.68)^{28}$ for longitudinal studies; while for cross-sectional studies the unadjusted ORs ranged from $0.8(95 \% \mathrm{CI}=0.4 ; 1.5)^{25}$ to 2.17 $(95 \% \mathrm{CI}=1.26 ; 3.73)^{22}$ and the adjusted ORs ranged from $1.04(95 \% \mathrm{CI}=1.02 ; 1.07)^{21}$ to $7.16(95 \% \mathrm{CI}=2.34 ; 21.89){ }^{20}$ Dias et al's ${ }^{26}$ study did not present the OR values. Of the five studies that presented the adjusted OR, ${ }^{19-21,24,28}$ one study did not report the adjustment variables. ${ }^{19}$ Among the adjustment variables reported, the most common were sex, age, comorbidities, history of falls in the last year, and body mass index.

Table 2 shows the quality analysis for longitudinal studies. All studies scored 5 points, indicating a moderate risk of bias. The study by Kamide et $\mathrm{al}^{24}$ lost points because it did not present the selection of the unexposed cohort, had a short follow-up period ( 1 month), and presented a sample loss of about $50 \%$ of the participants. The study by Curcio et $\mathrm{al}^{23}$ scored 5 points because the authors did not describe the selection of the unexposed cohort, did not use adjustment variables in the data analysis, and presented a sample loss of about $20 \%$ of the participants. Arfken et al's ${ }^{28}$ study did not inform the selection of the unexposed cohort, did not state whether there was sample loss over the follow-up period, and did not use a standardized scale for assessing FoF.

Regarding cross-sectional studies, the quality of the studies ranged from 3 (high risk of bias) to 8 (low risk of bias). Öztürk et al's ${ }^{25}$ study scored 3 points, as it did not present inclusion criteria, the study setting and participants' characteristics were not described in detail, the exposure measurement was not reliably evaluated, and adjustment variables were not used. The studies by Akin et $\mathrm{al}^{27}$ and Dias et $\mathrm{al}^{26}$ scored 5, while the studies by Alcolea-Ruiz et al ${ }^{19}$

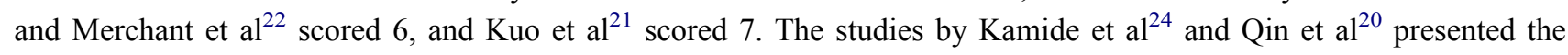
maximum score on the scale. More details on the methodological quality of included cross-sectional studies can be seen in Table 3.

\section{Discussion}

This study aimed to systematically review the available literature on the association between FoF (exposure) and frailty (outcome) in community-dwelling older adults. Most studies (longitudinal and cross-sectional) analyzed in this systematic review identified positive associations between the two geriatric syndromes. Specifically, two longitudinal and four cross-sectional studies presented significant adjusted ORs demonstrating an association between these variables. It is also noteworthy that most studies included in this review had a cross-sectional design and presented a considerable discrepancy in the methodological quality, with scores ranging from 3 (high risk of bias) to 8 (low risk of bias).

Regarding the longitudinal studies, adjusted ORs ranged from $1.18(95 \% \mathrm{CI}=1.02 ; 1.36)^{24}$ to $9.87(95 \% \mathrm{CI}=5.22$; $18.68){ }^{28}$ while adjusted ORs of the cross-sectional studies ranged from $1.04(95 \% \mathrm{CI}=1.02 ; 1.07)^{21}$ to $7.16(95 \%$ 
Table 3 Quality Assessment of Analytical Cross-Sectional Studies ( $=8$ ) Using the Joanna Briggs Institute (JBI) Critical Appraisal Checklist

\begin{tabular}{|c|c|c|c|c|c|c|c|c|c|}
\hline \multirow[t]{2}{*}{ Source } & \multicolumn{9}{|c|}{ Criteria } \\
\hline & $\begin{array}{l}\text { Were the } \\
\text { Criteria for } \\
\text { Inclusion in } \\
\text { the Sample } \\
\text { Clearly } \\
\text { Defined? }\end{array}$ & $\begin{array}{l}\text { Were the } \\
\text { Study } \\
\text { Subjects and } \\
\text { the Setting } \\
\text { Described in } \\
\text { Detail? }\end{array}$ & $\begin{array}{l}\text { Was the } \\
\text { Exposure } \\
\text { Measured } \\
\text { in a Valid } \\
\text { and } \\
\text { Reliable } \\
\text { Way? }\end{array}$ & $\begin{array}{c}\text { Were } \\
\text { Objective, } \\
\text { Standard } \\
\text { Criteria Used } \\
\text { for } \\
\text { Measurement } \\
\text { of the } \\
\text { Condition? }\end{array}$ & $\begin{array}{c}\text { Were } \\
\text { Confounding } \\
\text { Factors } \\
\text { Identified? }\end{array}$ & $\begin{array}{c}\text { Were } \\
\text { Strategies to } \\
\text { Address } \\
\text { Confounding } \\
\text { Factors } \\
\text { Stated? }\end{array}$ & $\begin{array}{l}\text { Were the } \\
\text { Outcomes } \\
\text { Measured in } \\
\text { a Valid and } \\
\text { Reliable } \\
\text { Way? }\end{array}$ & $\begin{array}{c}\text { Was } \\
\text { Appropriate } \\
\text { Statistical } \\
\text { Analysis } \\
\text { Used? }\end{array}$ & Total Score \\
\hline $\begin{array}{l}\text { Öztürk } \\
\text { et al. } \\
(2020)^{25}\end{array}$ & No & No & No & Yes & No & No & Yes & Yes & 3 \\
\hline $\begin{array}{l}\text { Alcolea- } \\
\text { Ruiz et al. } \\
(2021)^{19}\end{array}$ & Yes & Yes & Yes & Yes & No & Yes & No & Yes & 6 \\
\hline $\begin{array}{l}\text { Kuo et al. } \\
(2021)^{21}\end{array}$ & Yes & Yes & Yes & Yes & Yes & Yes & No & Yes & 7 \\
\hline $\begin{array}{l}\text { Qin et al. } \\
(2021)^{20}\end{array}$ & Yes & Yes & Yes & Yes & Yes & Yes & Yes & Yes & 8 \\
\hline $\begin{array}{l}\text { Kamide } \\
\text { et al. } \\
(2020)^{24}\end{array}$ & Yes & Yes & Yes & Yes & Yes & Yes & Yes & Yes & 8 \\
\hline $\begin{array}{l}\text { Merchant } \\
\text { et al. } \\
(2020)^{22}\end{array}$ & Yes & No & Yes & Yes & No & Yes & Yes & Yes & 6 \\
\hline $\begin{array}{l}\text { Akın et al. } \\
(2015)^{27}\end{array}$ & Yes & Yes & No & Yes & No & No & Yes & Yes & 5 \\
\hline $\begin{array}{l}\text { Dias et al. } \\
(2011)^{26}\end{array}$ & Yes & Yes & Yes & Yes & No & No & Yes & No & 5 \\
\hline
\end{tabular}

$\mathrm{CI}=2.34 ; 21.89) .{ }^{20}$ The large variation in the ORs observed among the included studies may be related to racial, demographic, anthropometric, socioeconomic, and cultural differences in the populations studied, being that four studies were conducted with participants from Europe, four from Asia, two from North America, and two from South America. Another issue involves the methodological aspects of the studies, such as different instruments used to assess frailty and FoF and different statistical analyses performed, which when adjusted considered different confounding variables. Furthermore, it should be noted that only the study by Öztürk et $\mathrm{al}^{25}$ found no significant association between the variables. According to the authors, the lack of association between these conditions is because common diseases in older adults, such as frailty and sarcopenia, have a bidirectional relationship with the FoF, generating a vicious cycle, which makes it difficult to establish an association. ${ }^{25}$

Among the ten studies analyzed in this systematic review, one study showed no $\mathrm{OR}^{26}{ }^{26}$ four studies presented only crude $\mathrm{OR},{ }^{22,23,25,27}$ one study presented adjusted OR without reporting the adjustment variables, ${ }^{19}$ and four studies presented adjusted OR with the adjustment variables..$^{20,21,24,28}$ This considerably limited the interpretation of the findings. Among the adjustment variables included in the studies, sex, age, comorbidities, history of falls in the past year, and body mass index were the most commonly used. MacKay et $\mathrm{al}^{30}{ }^{30}$ in a recent scoping review, observed that female sex, history of falls, low physical performance, and depressive symptoms were the factors most associated with FoF. This finding reinforces the importance of including these variables in association models encompassing the FoF variable. 
The mechanisms by which FoF can lead to frailty in older adults have been widely studied. ${ }^{19,21-27,31}$ Among them, it should be noted that older adults with FoF may present exacerbated concern about suffering falls, causing self-imposed restriction in performing activities of daily living, ${ }^{32}$ which in turn leads in the long term to decline in physical abilities (eg, reduced muscle strength, balance and mobility deficits, and physical deconditioning), thus increasing the risk for the development of other negative health conditions, such as sarcopenia and frailty. ${ }^{22}$ Corroborating this hypothesis, Auais et a ${ }^{33}$ showed that a one-point increase in the FES-I scale was associated with a $4 \%$ higher risk of mobility disability $(95 \% \mathrm{CI}=1.02 ; 1.05)$ and a $3 \%$ higher risk of developing poor physical performance $(95 \% \mathrm{CI}=1.01 ; 1.05)$ after a two-year follow-up. The authors pointed that reduced mobility, as well as poor physical performance, may be predisposing factors for frailty.

Previous study has also shown that self-imposed restriction in activities of daily living due to FoF is related to decreased energy expenditure in older adults, contributing to intramuscular fat accumulation. ${ }^{32}$ Intramuscular adipose tissue acts in the release of pro-inflammatory cytokines, such as TNF- $\alpha$, IL-6, C-reactive protein, as well as promoting increased levels of leukocytes and fibrinogen, which contributes to inflammatory conditions involved in the frailty etiopathogenesis. ${ }^{34,35}$ Increased levels of pro-inflammatory cytokines further contribute to muscle protein degradation and affect metabolic pathways important in regulating homeostasis, which results in reduced muscle strength and proprioception, favoring the onset of frailty in older adults. ${ }^{36}$ Moreover, Rochat et $\mathrm{al}^{37}$ demonstrated that FoF-related activity restriction is associated with changes in gait, such as reduced speed, which is one of the frailty phenotype criteria proposed by Fried et al. ${ }^{29}$

Although the evidence in the present review is not unanimous about the association between FoF and frailty, the results of this study demonstrate the importance of early identification of older adults with FoF, because this geriatric syndrome would be a factor associated with frailty. Although there are different screening tools to identify the frailty syndrome, some instruments have been most frequently used in clinical research, such as the Fried et al's ${ }^{29}$ scale. ${ }^{22,23,25-27}$ However, other tools can also be used, such as the Tilburg Frailty Indicator ${ }^{20}$ and the Short Physical Performance Battery. ${ }^{19}$ The Tilburg Frailty Indicator is a multidimensional questionnaire of frailty assessment that has higher reliability and validity compared to 38 instruments used to assess frailty, including the Fried et al's ${ }^{29}$ scale. ${ }^{38}$ The Short Physical Performance Battery, on the other hand, is an easy-to-apply tool to verify older adults' functional capacity and is considered by some authors as just a complementary tool in tracking frailty in older adults. ${ }^{39}$

The differences in definitions of frailty and FoF, assessment tools, participants' characteristics, and statistical methods made it impossible to perform pooled estimates (ie, meta-analysis). Another limiting aspect concerns the absence of the OR in the study by Dias et al, ${ }^{26}$ which may have been different from the range observed in other studies. In addition, most of the evidence included in this review comes from cross-sectional studies. Thus, the direction of the associations (ie, causality) cannot be determined.

As for the strengths of this study, we highlight the broad literature search in several databases, which increased the chance of identifying relevant studies in this field of knowledge, as well as the comprehensiveness of the sampling, including participants from different continents, cultures, and health perception, enabling a global view of the association between these two geriatric syndromes. Moreover, our systematic review provides for the first time an overview of the available evidence on the association between FoF and frailty in community-dwelling older adults. It is recommended that future research be conducted to determine the association between frailty (exposure) and FoF (outcome), as the literature suggests the existence of a bidirectional relationship between the two conditions. ${ }^{40}$ Future research should also present crude and adjusted ORs in order to reduce the risk of bias. Furthermore, further longitudinal research correcting the methodological shortcomings presented in the studies included in this review is highly recommended.

\section{Conclusion}

Longitudinal and cross-sectional evidence shows that FoF is associated with frailty in community-dwelling older adults. The knowledge of this association is important for clinical practice to assist health professionals in the development of early screening protocols for frailty, as well as rehabilitation, prevention, and health promotion strategies. Furthermore, it can contribute to prevention and health education programs aimed at reducing the FoF and, consequently, the development of frailty and its negative outcomes. 


\section{Disclosure}

The authors report no conflicts of interest in this work.

\section{References}

1. Collard RM, Boter H, Schoevers RA, Oude Voshaar RC. Prevalence of frailty in community-dwelling older persons: a systematic review. $J$ Am Geriatr Soc. 2012;60(8):1487-1492. doi:10.1111/j.1532-5415.2012.04054.x

2. Siriwardhana DD, Hardoon S, Rait G, Weerasinghe MC, Walters KR. Prevalence of frailty and prefrailty among community-dwelling older adults in low-income and middle-income countries: a systematic review and meta-analysis. BMJ Open. 2018;8(3):e018195. doi:10.1136/bmjopen-2017018195

3. Venturini C, Sampaio RF, de Souza Moreira B, et al. A multidimensional approach to frailty compared with physical phenotype in older Brazilian adults: data from the FIBRA-BR study. BMC Geriatr. 2021;21(1):246. doi:10.1186/s12877-021-02193-y

4. Chu W, Chang S-F, Ho H-Y. Adverse health effects of frailty: systematic review and meta-analysis of middle-aged and older adults with implications for evidence-based practice. Worldviews Evid Based Nurs. 2021;18(4):282-289. doi:10.1111/wvn.12508

5. Lee H, Lee E, Jang IY. Frailty and comprehensive geriatric assessment. J Korean Med Sci. 2020;35(3):e16. doi:10.3346/jkms.2020.35.e16

6. Fried LP, Ferrucci L, Darer J, Williamson JD, Anderson G. Untangling the concepts of disability, frailty, and comorbidity: implications for improved targeting and care. J Gerontol a Biol Sci Med Sci. 2004;59(3):255-263. doi:10.1093/gerona/59.3.m255

7. Chang S-F, Lin P-L. Frail phenotype and mortality prediction: a systematic review and meta-analysis of prospective cohort studies. Int $J$ Nurs Stud. 2015;52(8):1362-1374. doi:10.1016/j.ijnurstu.2015.04.005

8. Hoogendijk EO, Afilalo J, Ensrud KE, Kowal P, Onder G, Fried LP. Frailty: implications for clinical practice and public health. Lancet. 2019;394 (10206):1365-1375. doi:10.1016/S0140-6736(19)31786-6

9. Feng Z, Lugtenberg M, Franse C, et al. Risk factors and protective factors associated with incident or increase of frailty among communitydwelling older adults: a systematic review of longitudinal studies. PLoS One. 2017;12(6):e0178383. doi:10.1371/journal.pone.0178383

10. Tinetti ME, Richman D, Powell L. Falls efficacy as a measure of fear of falling. J Gerontol. 1990;45(6):239-243. doi:10.1093/geronj/45.6.P239

11. Kumar A, Carpenter H, Morris R, Iliffe S, Kendrick D. Which factors are associated with fear of falling in community-dwelling older people? Age Ageing. 2014;43(1):76-84. doi:10.1093/ageing/aft154

12. de Souza Moreira B, Sampaio RF, Furtado SRC, et al. The relationship between diabetes mellitus, geriatric syndromes, physical function, and gait: a review of the literature. Curr Diabetes Rev. 2016;12(3):240-251. doi:10.2174/1573399811666150615142746

13. Delbaere K, Crombez G, Vanderstraeten G, Willems T, Cambier D. Fear-related avoidance of activities, falls and physical frailty. A prospective community-based cohort study. Age Ageing. 2004;33(4):368-373. doi:10.1093/ageing/afh106

14. Belloni G, Büla C, Santos-Eggimann B, Henchoz Y, Fustinoni S, Seematter-Bagnoud L. Fear of falling and all-cause mortality among young-old community-dwelling adults: a 6-year prospective study. Eur J Ageing. 2021. doi:10.1007/s10433-021-00635-5

15. Kim J-H, Bae SM. Association between Fear of Falling (FOF) and all-cause mortality. Arch Gerontol Geriatr. 2020;88:104017. doi:10.1016/j. archger.2020.104017

16. Scheffer AC, Schuurmans MJ, Van dijk N, Van der Hooft T, De Rooij SE. Fear of falling: measurement strategy, prevalence, risk factors and consequences among older persons. Age Ageing. 2008;37(1):19-24. doi:10.1093/ageing/afm169

17. Stang A. Critical evaluation of the Newcastle-Ottawa scale for the assessment of the quality of nonrandomized studies in meta-analyses. Eur $J$ Epidemiol. 2010;25(9):603-605. doi:10.1007/s10654-010-9491-z

18. Santos dos WM, Secoli SR, Püschel VA de A. The Joanna Briggs Institute approach for systematic reviews. Rev Lat Am Enfermagem. $2018 ; 26: 45$.

19. Alcolea-Ruiz A-RS, Esteban-Paredes F, Beamud-Lagos M, Villar-Espejo MT, Pérez-Rivas FJ. [Prevalence of fear of falling and related factors in community-dwelling older people]. Aten Primaria. 2021;53(2):101962. Romanian. doi:10.1016/j.aprim.2020.11.003

20. Qin Y, Li J, McPhillips M, Lukkahatai N, Yu F, Li K. Association of fear of falling with frailty in community-dwelling older adults: a crosssectional study. Nurs Health Sci. 2021;23(2):516-524. doi:10.1111/nhs.12840

21. Kuo C-T, Chen D-R, Chen Y-M, Chen P-Y. Validation of the short falls efficacy scale-international for Taiwanese community-dwelling older adults: associations with fall history, physical frailty, and quality of life. Geriatr Nurs. 2021;42(5):1012-1018. doi:10.1016/j.gerinurse.2021.06.006

22. Merchant RA, Chen MZ, Wong BLL, et al. Relationship between fear of falling, fear-related activity restriction, frailty, and sarcopenia. $J A m$ Geriatr Soc. 2020;68(11):2602-2608. doi:10.1111/jgs.16719

23. Curcio C-L, Wu YY, Vafaei A, et al. A regression tree for identifying risk factors for fear of falling: the International Mobility in Aging Study (IMIAS). J Gerontol a Biol Sci Med Sci. 2020;75(1):181-188. doi:10.1093/gerona/glz002

24. Kamide N, Inoue N, Sakamoto M, Sato H, Shiba Y. [Fall-related efficacy is associated with the progression of frailty in community-dwelling older people]. Nihon Ronen Igakkai Zasshi. 2020;57(3):308-315. Japanese. doi:10.3143/geriatrics.57.308

25. Öztürk BG, Kılıç C, Bozkurt ME, Karan MA. Prevalence and associates of fear of falling among community-dwelling older adults. $J$ Nutr Health Aging. 2021;25(4):433-439. doi:10.1007/s12603-020-1535-9

26. Dias RC, Freire MTF, Santos ÉGS, Vieira RA, Dias JMD, Perracini MR. Characteristics associated with activity restriction induced by fear of falling in community-dwelling elderly. Braz J Phys Ther. 2011;15(5):406-413.

27. Akın S, Mazıcioglu M, Mucuk S, et al. The prevalence of frailty and related factors in community-dwelling Turkish elderly according to modified Fried Frailty Index and FRAIL scales. Aging Clin Exp Res. 2015;27(5):703-709. doi:10.1007/s40520-015-0337-0

28. Arfken CL, Lach HW, Birge SJ, Miller JP. The prevalence and correlates of fear of falling in elderly persons living in the community. Am J Public Health. 1994;84(4):565-570. doi:10.2105/ajph.84.4.565

29. Fried LP, Young Y, Rubin G, Bandeen-Roche K. Self-reported preclinical disability identifies older women with early declines in performance and early disease. J Clin Epidemiol. 2001;54(9):889-901. doi:10.1016/s0895-4356(01)00357-2

30. MacKay S, Ebert P, Harbidge C, Hogan DB. Fear of falling in older adults: a scoping review of recent literature. Can Geriatr J. 2021;24(4):379394. doi: $10.5770 /$ cgj.24.521

31. Qin W, Xu L, Sun L, et al. Association between frailty and life satisfaction among older people in Shandong, China: the differences in age and general self-efficacy. Psychogeriatrics. 2020;20(2):172-179. doi:10.1111/psyg.12482 
32. Lavedán A, Viladrosa M, Jürschik P, et al. Fear of falling in community-dwelling older adults: a cause of falls, a consequence, or both? PLoS One. 2018;13(3):1-14. doi:10.1371/journal.pone.0194967

33. Auais M, French S, Alvarado B, Pirkle C, Belanger E, Guralnik J. Fear of falling predicts incidence of functional disability 2 years later: a perspective from an international cohort study. J Gerontol A. 2018;73(9):1212-1215. doi:10.1093/gerona/glx237

34. Soysal P, Stubbs B, Lucato P, et al. Inflammation and frailty in the elderly: a systematic review and meta-analysis. Ageing Res Rev. 2016;31:1-8. doi:10.1016/j.arr.2016.08.006

35. Addison O, Drummond MJ, LaStayo PC, et al. Intramuscular fat and inflammation differ in older adults: the impact of frailty and inactivity. $J$ Nutr Health Aging. 2014;18(5):532-538. doi:10.1007/s12603-014-0019-1

36. Lang P-O, Michel J-P, Zekry D. Frailty syndrome: a transitional state in a dynamic process. Gerontology. 2009;55(5):539-549. doi:10.1159/ 000211949

37. Rochat S, Büla CJ, Martin E, et al. What is the relationship between fear of falling and gait in well-functioning older persons aged 65 to 70 years? Arch Phys Med Rehabil. 2010;91(6):879-884. doi:10.1016/j.apmr.2010.03.005

38. Robbert JJ. The Tilburg frailty indicator: psychometric properties. J Am Med Dir Assoc. 2010;11(5):344-355. doi:10.1016/j.jamda.2009.11.003

39. Ramírez-Vélez R, López Sáez de Asteasu M, Morley JE, Cano-Gutierrez CA, Izquierdo M. Performance of the short physical performance battery in identifying the frailty phenotype and predicting geriatric syndromes in community-dwelling elderly. J Nutr Health Aging. 2021;25(2):209-217. doi:10.1007/s12603-020-1484-3

40. Wijlhuizen GJ, Chorus AMJ, Hopman-Rock M. Fragility, fear of falling, physical activity and falls among older persons: some theoretical considerations to interpret mediation. Prev Med. 2008;46(6):612-614. doi:10.1016/j.ypmed.2008.01.016

41. Page MJ, McKenzie JE, Bossuyt PM, et al. The PRISMA 2020 statement: an updated guideline for reporting systematic reviews. BMJ. 2021;372: n71. doi:10.1136/bmj.n71

\section{Publish your work in this journal}

Clinical Interventions in Aging is an international, peer-reviewed journal focusing on evidence-based reports on the value or lack thereof of treatments intended to prevent or delay the onset of maladaptive correlates of aging in human beings. This journal is indexed on PubMed Central, MedLine, CAS, Scopus and the Elsevier Bibliographic databases. The manuscript management system is completely online and includes a very quick and fair peer-review system, which is all easy to use. Visit http://www.dovepress.com/testimonials.php to read real quotes from published authors.

Submit your manuscript here: https://www.dovepress.com/clinical-interventions-in-aging-journal 